(C) 2011 IEEE. Personal use of this material is permitted. Permission from IEEE must be obtained for all other uses, in any current or future media, including reprinting/republishing this material for advertising or promotional purposes, creating new collective works, for resale or redistribution to servers or lists, or reuse of any copyrighted component of this work in other works. 


\title{
Robust Design for Linear Non-Regenerative MIMO Relays with Imperfect Channel State Information
}

\author{
Yue Rong, Member, IEEE
}

\begin{abstract}
In this paper, we address statistically robust multiple-input multiple-output (MIMO) relay design problems under two imperfect channel state information (CSI) scenarios: (1) All nodes have imperfect CSI; (2) The destination node knows the exact CSI, while the other nodes have imperfect CSI. For each scenario, we develop robust source and relay matrices by considering a broad class of frequently used objective functions in MIMO system design and the averaged transmission power constraints. Simulation results demonstrate the improved robustness of the proposed algorithms against CSI errors.
\end{abstract}

Index Terms-MIMO relay, channel state information, robustness, MMSE, majorization.

\section{INTRODUCTION}

Recently, there have been many research efforts on linear non-regenerative multiple-input multiple-output (MIMO) relay systems [1]-[4]. The optimal relay amplifying matrix is obtained in [1], [2] to maximize the mutual information between source and destination. In [3], optimal relay matrices are developed to minimize the mean-squared error (MSE) of the signal waveform estimation at the destination. A unified framework is established for optimizing the source precoding matrix and the relay amplifying matrix of linear non-regenerative MIMO relay systems with a broad class of objective functions [4].

For MIMO relay systems, the channel state information (CSI) knowledge of all hops is required at the destination node to estimate the source signals. Moreover, in order to optimize the source and relay matrices in [1]-[4], the CSI knowledge of all hops is needed at the node which carries out the optimization procedure. However, in practical relay communication systems, the exact CSI is unknown, and therefore, has to be estimated. There is always mismatch between the true and the estimated CSI due to channel noise, quantization errors, and outdated channel estimates. Obviously, the performance of the algorithms in [1]-[4] will degrade due to such CSI mismatch. In [5]-[7], MMSE-based optimal relay amplifying and destination receiving matrices for a two-hop MIMO relay system have been developed taking into account the CSI mismatch. However, the source precoding matrix is not optimized in [5]-[7]. The source precoding matrix optimization under CSI mismatch is investigated in [8], [9] using the MMSE criterion.

In this paper, we investigate statistically robust two-hop MIMO relay systems. In contrast to [5]-[9], we develop

Yue Rong is with the Department of Electrical and Computer Engineering, Curtin University, Bentley, WA 6102, Australia. E-mail: y.rong @ curtin.edu.au. This paper was presented in part at AusCTW, Canberra, Australia, Feb. 2010.

This work was supported under Australian Research Council's Discovery Projects funding scheme (project numbers DP110100736, DP110102076). robust source precoding matrix and relay amplifying matrix by considering a broad class of frequently used objective functions in MIMO system design [4] (e.g. maximal mutual information, MMSE). In particular, we consider two imperfect CSI scenarios: (1) All nodes have imperfect CSI; (2) The destination node knows the exact CSI, while the source and relay nodes have imperfect CSI. The true CSI is modelled as a Gaussian random matrix with the estimated CSI as the mean value, and the well-known Kronecker model is adopted for the covariance of the CSI mismatch [5]-[9]. We would like to point out that in [10], only the imperfect CSI case (1) is addressed.

For each of the two imperfect CSI scenarios, we show that the CSI mismatch information is intrinsically embedded in the structure of the optimal robust source and relay matrices. Moreover, the available power at the source and the relay nodes is optimally distributed among all data streams in a robust fashion against the CSI mismatch. Such robust power allocation can be implemented with the same computational complexity as the non-robust power allocation scheme in [4]. Interestingly, when the exact CSI is available (i.e., no CSI mismatch), the robust source and relay matrices become the optimal source and relay matrices developed in [4]. Thus, this paper is an important generalization of [4] to the practical scenario of imperfect CSI. Simulation results demonstrate the improved robustness of the proposed approaches against the CSI mismatch.

The rest of this paper is organized as follows. In Section II, we introduce the model of a two-hop linear non-regenerative MIMO relay communication system. The robust source and relay matrices are developed in Sections III and IV, depending on whether the destination node has the exact CSI knowledge. In Section V, we show some numerical examples. Conclusions are drawn in Section VI.

\section{SySTEM MODEL}

We consider a three-node MIMO communication system where the source node (node 1) transmits information to the destination node (node 3 ) with the aid of one relay node (node 2 ). The $i$ th node is equipped with $N_{i}, i=1,2,3$, antennas. We focus on the case where the direct link between the source and destination nodes is sufficiently weak to be ignored as in [1][4]. This scenario occurs when the direct link is blocked by an obstacle such as a mountain. Using the non-regenerative relay strategy, the received signal vector at the destination node can be written as

$$
\mathbf{y}=\mathbf{H}_{2} \mathbf{F}_{2} \mathbf{H}_{1} \mathbf{F}_{1} \mathbf{s}+\mathbf{H}_{2} \mathbf{F}_{2} \mathbf{v}_{2}+\mathbf{v}_{3} \triangleq \mathbf{G s}+\overline{\mathbf{v}}
$$


where $\mathbf{s}$ is the $N_{b} \times 1$ source signal vector, $\mathbf{F}_{1}$ is the $N_{1} \times N_{b}$ source precoding matrix, $\mathbf{H}_{1}$ is the $N_{2} \times N_{1}$ MIMO fading channel matrix between the source and relay nodes, $\mathbf{F}_{2}$ is the $N_{2} \times N_{2}$ relay amplifying matrix, $\mathbf{H}_{2}$ is the $N_{3} \times N_{2}$ MIMO fading channel matrix between the relay and destination nodes, $\mathbf{v}_{2}$ is an $N_{2} \times 1$ noise vector at the relay node, and $\mathbf{v}_{3}$ is an $N_{3} \times 1$ noise vector at the destination node. Here $\mathbf{G} \triangleq \mathbf{H}_{2} \mathbf{F}_{2} \mathbf{H}_{1} \mathbf{F}_{1}$ is the equivalent source-destination MIMO channel matrix, and $\overline{\mathbf{v}} \triangleq \mathbf{H}_{2} \mathbf{F}_{2} \mathbf{v}_{2}+\mathbf{v}_{3}$ is the equivalent noise vector.

We assume that $\mathrm{E}\left[\mathbf{s s}^{H}\right]=\mathbf{I}_{N_{b}}$, where $\mathrm{E}[\cdot]$ stands for the statistical expectation, $(\cdot)^{H}$ denotes the Hermitian transpose, and $\mathbf{I}_{n}$ is an $n \times n$ identity matrix. In order to avoid any transmission power loss at each node, there should be $N_{b} \leq \min \left(N_{1}, N_{2}, N_{3}\right)$. We also assume that all noises are independent and identically distributed (i.i.d.) additive white Gaussian noise (AWGN) with zero mean and unit variance.

Using a linear receiver at the destination node, the estimated signal vector can be written as $\hat{\mathbf{s}}=\mathbf{W}^{H} \mathbf{y}$, where $\mathbf{W}$ is the $N_{3} \times N_{b}$ weight matrix. The MSE matrix of the signal waveform estimation $\mathbf{E} \triangleq \mathrm{E}\left[(\hat{\mathbf{s}}-\mathbf{s})(\hat{\mathbf{s}}-\mathbf{s})^{H}\right]$ can be written as

$$
\mathbf{E}=\left(\mathbf{W}^{H} \mathbf{G}-\mathbf{I}_{N_{b}}\right)\left(\mathbf{W}^{H} \mathbf{G}-\mathbf{I}_{N_{b}}\right)^{H}+\mathbf{W}^{H} \mathbf{C}_{\bar{v}} \mathbf{W}
$$

where $\mathbf{C}_{\bar{v}} \triangleq \mathrm{E}\left[\overline{\mathbf{v}} \overline{\mathbf{v}}^{H}\right]=\mathbf{H}_{2} \mathbf{F}_{2} \mathbf{F}_{2}^{H} \mathbf{H}_{2}^{H}+\mathbf{I}_{N_{3}}$ is the noise covariance matrix. It has been shown in [4] that a broad class of frequently used MIMO relay system design objectives such as the source-destination mutual information can be written as a function of the main diagonal elements of the MSE matrix E.

With mismatch between the true and the estimated CSI, the true channel $\mathbf{H}_{i}$ can be represented by the well-known Gaussian-Kronecker model [5]-[9], where $\mathbf{H}_{i}$ is a complexvalued Gaussian random matrix

$$
\mathbf{H}_{i} \sim \mathcal{C N}\left(\overline{\mathbf{H}}_{i}, \boldsymbol{\Theta}_{i} \otimes \boldsymbol{\Phi}_{i}\right), \quad i=1,2 .
$$

Here the mean value is the estimated channel matrix $\overline{\mathbf{H}}_{i}, \boldsymbol{\Theta}_{i}$ denotes the $N_{i} \times N_{i}$ covariance matrix of channel estimation error at the transmitter side, while $\boldsymbol{\Phi}_{i}$ is the $N_{i+1} \times N_{i+1}$ covariance matrix of channel estimation error seen from the receiver side, and $\otimes$ stands for the matrix Kronecker product. In other words, we have $\mathbf{H}_{i}=\overline{\mathbf{H}}_{i}+\mathbf{A}_{\Phi_{i}} \mathbf{H}_{\mathrm{w}_{i}} \mathbf{A}_{\Theta_{i}}^{H}, i=1,2$, where $\mathbf{A}_{\Phi_{i}} \mathbf{A}_{\Phi_{i}}^{H}=\boldsymbol{\Phi}_{i}, \mathbf{A}_{\Theta_{i}} \mathbf{A}_{\Theta_{i}}^{H}=\boldsymbol{\Theta}_{i}^{T}$, and $\mathbf{H}_{\mathrm{w}_{i}}$ is an $N_{i+1} \times$ $N_{i}$ Gaussian random matrix with i.i.d. zero mean and unit variance entries, and is the unknown part in the CSI mismatch.

LEMMA 1 [12]: For $\mathbf{H} \sim \mathcal{C N}(\overline{\mathbf{H}}, \Theta \otimes \boldsymbol{\Phi})$, there is $\mathrm{E}_{\mathrm{H}}\left[\mathbf{H} \mathbf{A} \mathbf{H}^{H}\right]=\overline{\mathbf{H}} \mathbf{A} \overline{\mathbf{H}}^{H}+\operatorname{tr}\left(\mathbf{A} \boldsymbol{\Theta}^{T}\right) \boldsymbol{\Phi}$, and $\mathrm{E}_{\mathrm{H}}\left[\mathbf{H}^{H} \mathbf{A} \mathbf{H}\right]=$ $\overline{\mathbf{H}}^{H} \mathbf{A} \overline{\mathbf{H}}+\operatorname{tr}(\mathbf{\Phi} \mathbf{A}) \boldsymbol{\Theta}^{T}$, where $\operatorname{tr}(\cdot)$ denotes the matrix trace, and $(\cdot)^{T}$ stands for the matrix transpose.

\section{Robust MiMO Relay Design with Imperfect CSI AT All NODES}

In this section, we consider the scenario where all nodes have imperfect CSI. It can be seen from (2) that if the exact $\mathbf{H}_{1}$ and $\mathbf{H}_{2}$ are unavailable at the destination node, it is impossible to design $\mathbf{W}$ that optimizes $\mathbf{E}$ in (2). If we design $\mathbf{W}, \mathbf{F}_{1}$, and $\mathbf{F}_{2}$ based only on $\overline{\mathbf{H}}_{1}$ and $\overline{\mathbf{H}}_{2}$, there can be a great performance degradation due to the mismatch between $\mathbf{H}_{i}$ and $\overline{\mathbf{H}}_{i}, i=1,2$. Instead of optimizing $\mathbf{E}$, we design $\mathbf{W}$ to minimize $\mathrm{E}_{\mathrm{H}_{1}, \mathrm{H}_{2}}[\mathbf{E}]$, where the statistical expectation is carried out with respect to $\mathbf{H}_{1}$ and $\mathbf{H}_{2}$, with the distribution given in (3).

The statistical expectation of $\mathbf{E}$ is given by [10]

$$
\begin{array}{r}
\mathrm{E}_{\mathrm{H}_{1}, \mathrm{H}_{2}}[\mathbf{E}]=\mathbf{W}^{H} \mathbf{A W}-\mathbf{W}^{H} \overline{\mathbf{H}}_{2} \mathbf{F}_{2} \overline{\mathbf{H}}_{1} \mathbf{F}_{1} \\
-\mathbf{F}_{1}^{H} \overline{\mathbf{H}}_{1}^{H} \mathbf{F}_{2}^{H} \overline{\mathbf{H}}_{2}^{H} \mathbf{W}+\mathbf{I}_{N_{b}}
\end{array}
$$

where

$$
\begin{aligned}
\mathbf{A} \triangleq & \overline{\mathbf{H}}_{2} \mathbf{F}_{2}\left(\overline{\mathbf{H}}_{1} \mathbf{F}_{1} \mathbf{F}_{1}^{H} \overline{\mathbf{H}}_{1}^{H}+\alpha_{1} \mathbf{\Phi}_{1}+\mathbf{I}_{N_{2}}\right) \mathbf{F}_{2}^{H} \overline{\mathbf{H}}_{2}^{H} \\
& +\alpha_{2} \mathbf{\Phi}_{2}+\mathbf{I}_{N_{3}} \\
\alpha_{1} \triangleq & \operatorname{tr}\left(\mathbf{F}_{1} \mathbf{F}_{1}^{H} \mathbf{\Theta}_{1}^{T}\right) \\
\alpha_{2} \triangleq & \operatorname{tr}\left(\mathbf{F}_{2}\left(\overline{\mathbf{H}}_{1} \mathbf{F}_{1} \mathbf{F}_{1}^{H} \overline{\mathbf{H}}_{1}^{H}+\alpha_{1} \boldsymbol{\Phi}_{1}+\mathbf{I}_{N_{2}}\right) \mathbf{F}_{2}^{H} \mathbf{\Theta}_{2}^{T}\right) .
\end{aligned}
$$

Now the weight matrix $\mathbf{W}$ which minimizes (4) is the famous Wiener filter given by

$$
\mathbf{W}=\mathbf{A}^{-1} \overline{\mathbf{H}}_{2} \mathbf{F}_{2} \overline{\mathbf{H}}_{1} \mathbf{F}_{1}
$$

where $(\cdot)^{-1}$ denotes the matrix inversion. Substituting (8) back into (4), we have

$$
\mathrm{E}_{\mathrm{H}_{1}, \mathrm{H}_{2}}[\mathbf{E}]=\mathbf{I}_{N_{b}}-\mathbf{F}_{1}^{H} \overline{\mathbf{H}}_{1}^{H} \mathbf{F}_{2}^{H} \overline{\mathbf{H}}_{2}^{H} \mathbf{A}^{-1} \overline{\mathbf{H}}_{2} \mathbf{F}_{2} \overline{\mathbf{H}}_{1} \mathbf{F}_{1} .
$$

The transmission power consumed by the relay node can be written as $p_{2}=\operatorname{tr}\left(\mathbf{F}_{2}\left(\mathbf{H}_{1} \mathbf{F}_{1} \mathbf{F}_{1}^{H} \mathbf{H}_{1}^{H}+\mathbf{I}_{N_{2}}\right) \mathbf{F}_{2}^{H}\right)$. However, since the true $\mathbf{H}_{1}$ is unknown, $p_{2}$ is also unknown. In this paper, we consider the averaged transmission power at the relay node, which is given by

$$
\begin{aligned}
\mathrm{E}_{\mathrm{H}_{1}}\left[p_{2}\right] & =\operatorname{tr}\left(\mathbf{F}_{2}\left(\mathrm{E}\left[\mathbf{H}_{1} \mathbf{F}_{1} \mathbf{F}_{1}^{H} \mathbf{H}_{1}^{H}\right]+\mathbf{I}_{N_{2}}\right) \mathbf{F}_{2}^{H}\right) \\
& =\operatorname{tr}\left(\mathbf{F}_{2}\left(\overline{\mathbf{H}}_{1} \mathbf{F}_{1} \mathbf{F}_{1}^{H} \overline{\mathbf{H}}_{1}^{H}+\alpha_{1} \mathbf{\Phi}_{1}+\mathbf{I}_{N_{2}}\right) \mathbf{F}_{2}^{H}\right)(10)
\end{aligned}
$$

where Lemma 1 is applied to obtain (10). As in [4], we use $q$ to denote a unified objective function, and $\mathbf{d}[\mathbf{E}]$ stands for the main diagonal elements of $\mathbf{E}$. Instead of optimizing $q(\mathbf{d}[\mathbf{E}])$ in [4], we minimize the objective function of $q\left(\mathbf{d}\left[\mathrm{E}_{\mathrm{H}_{1}, \mathrm{H}_{2}}[\mathbf{E}]\right]\right)$. Combining (9) and (10), the robust source and relay matrices optimization problem can be written as

$$
\begin{array}{rl}
\min _{\mathbf{F}_{1}, \mathbf{F}_{2}} & q\left(\mathbf{d}\left[\mathbf{I}_{N_{b}}-\mathbf{F}_{1}^{H} \overline{\mathbf{H}}_{1}^{H} \mathbf{F}_{2}^{H} \overline{\mathbf{H}}_{2}^{H} \mathbf{A}^{-1} \overline{\mathbf{H}}_{2} \mathbf{F}_{2} \overline{\mathbf{H}}_{1} \mathbf{F}_{1}\right]\right) \\
\text { s.t. } & \operatorname{tr}\left(\mathbf{F}_{2}\left(\overline{\mathbf{H}}_{1} \mathbf{F}_{1} \mathbf{F}_{1}^{H} \overline{\mathbf{H}}_{1}^{H}+\alpha_{1} \boldsymbol{\Phi}_{1}+\mathbf{I}_{N_{2}}\right) \mathbf{F}_{2}^{H}\right) \leq P_{2}(12 \\
& \operatorname{tr}\left(\mathbf{F}_{1} \mathbf{F}_{1}^{H}\right) \leq P_{1}
\end{array}
$$

where $P_{i}>0, i=1,2$, is the transmission power available at the $i$ th node, (12) and (13) represent the transmission power constraint at the relay node and the source node, respectively. The problem (11)-(13) provides a statistically robust design of $\mathbf{F}_{1}$ and $\mathbf{F}_{2}$ when all nodes have imperfect CSI.

Let us introduce the following matrix eigenvalue decomposition (EVD) and singular value decomposition (SVD) for $i=1,2$

$$
\begin{aligned}
\boldsymbol{\Phi}_{i} & =\mathbf{U}_{\Phi_{i}} \boldsymbol{\Lambda}_{\Phi_{i}} \mathbf{U}_{\Phi_{i}}^{H} \\
\tilde{\boldsymbol{\Lambda}}_{\Phi_{i}} & \triangleq \alpha_{i} \boldsymbol{\Lambda}_{\Phi_{i}}+\mathbf{I}_{N_{i+1}} \\
\tilde{\mathbf{H}}_{i} & \triangleq \tilde{\boldsymbol{\Lambda}}_{\Phi_{i}}^{-\frac{1}{2}} \mathbf{U}_{\Phi_{i}}^{H} \overline{\mathbf{H}}_{i}=\tilde{\mathbf{U}}_{i} \tilde{\boldsymbol{\Sigma}}_{i} \tilde{\mathbf{V}}_{i}^{H}
\end{aligned}
$$


where $\mathbf{U}_{\Phi_{i}}$ and $\tilde{\mathbf{U}}_{i}$ are $N_{i+1} \times N_{i+1}$ unitary matrices, $\boldsymbol{\Lambda}_{\Phi_{i}}$ is an $N_{i+1} \times N_{i+1}$ diagonal matrix, $\tilde{\mathbf{V}}_{i}$ is an $N_{i} \times N_{i}$ unitary matrix, and $\tilde{\boldsymbol{\Sigma}}_{i}$ is an $N_{i+1} \times N_{i}$ singularvalue matrix. It has been proven in [10] that for the statistically robust relay design problem (11)-(13), if $q$ is a Schur-concave function [11] of $\mathbf{d}\left[\mathrm{E}_{\mathrm{H}_{1}, \mathrm{H}_{2}}[\mathbf{E}]\right]$, the optimal $\mathbf{F}_{1}$ and $\mathbf{F}_{2}$ are given by

$$
\mathbf{F}_{1}=\tilde{\mathbf{V}}_{1,1} \boldsymbol{\Lambda}_{1}, \quad \mathbf{F}_{2}=\tilde{\mathbf{V}}_{2,1} \boldsymbol{\Lambda}_{2} \tilde{\mathbf{U}}_{1,1}^{H} \tilde{\boldsymbol{\Lambda}}_{\Phi_{1}}^{-\frac{1}{2}} \mathbf{U}_{\Phi_{1}}^{H}
$$

where for $i=1,2, \tilde{\mathbf{V}}_{i, 1}$ and $\tilde{\mathbf{U}}_{i, 1}$ corresponds to $N_{b}$ columns in $\tilde{\mathbf{V}}_{i}$ and $\tilde{\mathbf{U}}_{i}$ associated with the largest $N_{b}$ singularvalues, respectively, and $\boldsymbol{\Lambda}_{i}, i=1,2$, are $N_{b} \times N_{b}$ diagonal matrices. If $q$ is Schur-convex [11] with respect to $\mathbf{d}\left[\mathrm{E}_{\mathrm{H}_{1}, \mathrm{H}_{2}}[\mathbf{E}]\right]$, the optimal $\mathbf{F}_{2}$ is given in (17), while the optimal $\mathbf{F}_{1}$ is $\mathbf{F}_{1}=$ $\tilde{\mathbf{V}}_{1,1} \boldsymbol{\Lambda}_{1} \mathbf{V}_{0}$, where $\mathbf{V}_{0}$ is an $N_{b} \times N_{b}$ unitary matrix such that $\mathrm{E}_{\mathrm{H}_{1}, \mathrm{H}_{2}}[\mathbf{E}]$ in (9) has identical main-diagonal elements.

If the exact CSI is available at all nodes, i.e., $\mathbf{H}_{i}=\overline{\mathbf{H}}_{i}$, $\boldsymbol{\Theta}_{i}=\mathbf{0}_{N_{i}}, \boldsymbol{\Phi}_{i}=\mathbf{0}_{N_{i+1}}, i=1,2$, the problem (11)-(13) becomes the MIMO relay optimization problem with the exact CSI in [4]. Therefore, the problem (11)-(13) is more general than the problem in [4]. From (6) and (7) we find that $\alpha_{1}$ is a function of $\mathbf{F}_{1}$, and $\alpha_{2}$ is a function of both $\mathbf{F}_{1}$ and $\mathbf{F}_{2}$. Consequently, it can be seen from (14)-(16) that $\tilde{\mathbf{V}}_{1}$ and $\tilde{\mathbf{U}}_{1}$ depend on $\mathbf{F}_{1}$, and $\tilde{\mathbf{V}}_{2}$ depends on both $\mathbf{F}_{1}$ and $\mathbf{F}_{2}$. Thus, from (17) we find that the explicit structure of the optimal $\mathbf{F}_{1}$ and $\mathbf{F}_{2}$ is very difficult to find for general $\boldsymbol{\Theta}_{i}$ and $\boldsymbol{\Phi}_{i}$. In the following, we show the explicit structure of the optimal $\mathbf{F}_{1}$ and $\mathbf{F}_{2}$ when $\boldsymbol{\Theta}_{i}=\tau_{i} \mathbf{I}_{N_{i}}$ and/or $\boldsymbol{\Phi}_{i}=\varepsilon_{i} \mathbf{I}_{N_{i+1}}, i=1,2$. This corresponds to the MIMO channel where the transmit and/or receiver antennas are uncorrelated as explained in detail in Remark 1 of [7].

For the case of $\boldsymbol{\Phi}_{i}=\varepsilon_{i} \mathbf{I}_{N_{i+1}}, i=1,2$, the robust relay optimization problem can be written as

$$
\begin{array}{rl}
\min _{\mathbf{F}_{1}, \mathbf{F}_{2}} & q\left(\mathbf{d}\left[\mathbf{I}_{N_{b}}-\mathbf{F}_{1}^{H} \overline{\mathbf{H}}_{1}^{H} \mathbf{F}_{2}^{H} \overline{\mathbf{H}}_{2}^{H} \mathbf{B}^{-1} \overline{\mathbf{H}}_{2} \mathbf{F}_{2} \overline{\mathbf{H}}_{1} \mathbf{F}_{1}\right]\right) \\
\text { s.t. } & \operatorname{tr}\left(\mathbf{F}_{2}\left(\overline{\mathbf{H}}_{1} \mathbf{F}_{1} \mathbf{F}_{1}^{H} \overline{\mathbf{H}}_{1}^{H}+\beta_{1} \mathbf{I}_{N_{2}}\right) \mathbf{F}_{2}^{H}\right) \leq P_{2} \\
& \operatorname{tr}\left(\mathbf{F}_{1} \mathbf{F}_{1}^{H}\right) \leq P_{1}
\end{array}
$$

where

$$
\begin{aligned}
& \mathbf{B} \triangleq \overline{\mathbf{H}}_{2} \mathbf{F}_{2}\left(\overline{\mathbf{H}}_{1} \mathbf{F}_{1} \mathbf{F}_{1}^{H} \overline{\mathbf{H}}_{1}^{H}+\beta_{1} \mathbf{I}_{N_{2}}\right) \mathbf{F}_{2}^{H} \overline{\mathbf{H}}_{2}^{H}+\beta_{2} \mathbf{I}_{N_{3}} \\
& \beta_{1} \triangleq \varepsilon_{1} \operatorname{tr}\left(\mathbf{F}_{1} \mathbf{F}_{1}^{H} \mathbf{\Theta}_{1}^{T}\right)+1 \\
& \beta_{2} \triangleq \varepsilon_{2} \operatorname{tr}\left(\mathbf{F}_{2}\left(\overline{\mathbf{H}}_{1} \mathbf{F}_{1} \mathbf{F}_{1}^{H} \overline{\mathbf{H}}_{1}^{H}+\beta_{1} \mathbf{I}_{N_{2}}\right) \mathbf{F}_{2}^{H} \mathbf{\Theta}_{2}^{T}\right)+1
\end{aligned}
$$

Let us introduce the SVDs of $\overline{\mathbf{H}}_{i}=\mathbf{U}_{i} \boldsymbol{\Sigma}_{i} \mathbf{V}_{i}^{H}, i=1,2$. It can be easily seen from (14)-(16) that for $\boldsymbol{\Phi}_{i}=\varepsilon_{i} \mathbf{I}_{N_{i+1}}$, we have $\tilde{\mathbf{V}}_{i}=\mathbf{V}_{i}$ and $\tilde{\mathbf{U}}_{i}=\mathbf{U}_{i}, i=1,2$. Consequently, for Schur-concave $q$, we have

$$
\mathbf{F}_{1}=\mathbf{V}_{1,1} \boldsymbol{\Lambda}_{1}, \quad \mathbf{F}_{2}=\mathbf{V}_{2,1} \boldsymbol{\Lambda}_{2} \mathbf{U}_{1,1}^{H}
$$

where $\mathbf{V}_{i, 1}$ and $\mathbf{U}_{i, 1}$ corresponds to $N_{b}$ columns in $\mathbf{V}_{i}$ and $\mathbf{U}_{i}$ associated with the largest $N_{b}$ singularvalues, respectively. If $q$ is Schur-convex, $\mathbf{F}_{2}$ is given in (24), and the optimal $\mathbf{F}_{1}$ is $\mathbf{F}_{1}=\mathbf{V}_{1,1} \boldsymbol{\Lambda}_{1} \mathbf{V}_{0}$.

Now the task is to find the $N_{b} \times N_{b}$ diagonal power loading matrices $\boldsymbol{\Lambda}_{i}, i=1,2$. For Schur-concave $q$, substituting (24) back into (18)-(20), we obtain the following problem

$$
\begin{array}{ll}
\min _{\boldsymbol{\lambda}_{1}, \boldsymbol{\lambda}_{2}} & q\left(\left\{\left(1+\frac{\sigma_{1, k}^{2} \lambda_{1, k}^{2} \sigma_{2, k}^{2} \lambda_{2, k}^{2}}{\beta_{1} \sigma_{2, k}^{2} \lambda_{2, k}^{2}+\beta_{2}}\right)^{-1}\right\}\right) \\
\text { s.t. } & \sum_{k=1}^{N_{b}} \lambda_{2, k}^{2}\left(\lambda_{1, k}^{2} \sigma_{1, k}^{2}+\beta_{1}\right) \leq P_{2} \\
& \sum_{k=1}^{N_{b}} \lambda_{1, k}^{2} \leq P_{1} \\
& \lambda_{1, k} \geq 0, \quad \lambda_{2, k} \geq 0, \quad k=1, \cdots, N_{b}
\end{array}
$$

where $\beta_{1} \triangleq \varepsilon_{1} \sum_{k=1}^{N_{b}} \lambda_{1, k}^{2}\left[\mathbf{V}_{1,1}^{H} \Theta_{1}^{T} \mathbf{V}_{1,1}\right]_{k, k}+1, \beta_{2} \triangleq$ $\varepsilon_{2} \sum_{k=1}^{N_{b}} \lambda_{2, k}^{2}\left(\sigma_{1, k}^{2} \lambda_{1, k}^{2}+\beta_{1}\right)\left[\mathbf{V}_{2,1}^{H} \Theta_{2}^{T} \mathbf{V}_{2,1}\right]_{k, k}+1$. Here for $i=1,2, \lambda_{i, k}$ and $\sigma_{i, k}, k=1, \cdots, N_{b}$, are the $k$ th largest main diagonal elements of $\boldsymbol{\Lambda}_{i}$ and $\boldsymbol{\Sigma}_{i}$, respectively, $\boldsymbol{\lambda}_{i} \triangleq$ $\left[\lambda_{i, 1}, \cdots, \lambda_{i, N_{b}}\right]^{T}$, and for a scalar $x,\left\{x_{k}\right\} \triangleq\left[x_{1}, \cdots, x_{N_{b}}\right]^{T}$.

By introducing $a_{k} \triangleq \sigma_{1, k}^{2}, x_{k} \triangleq \lambda_{1, k}^{2}, b_{k} \triangleq \sigma_{2, k}^{2}, y_{k} \triangleq$ $\lambda_{2, k}^{2}\left(\lambda_{1, k}^{2} \sigma_{1, k}^{2}+\beta_{1}\right), k=1, \cdots, N_{b}$, the problem (25)-(28) can be simplified to

$$
\begin{array}{rl}
\min _{\mathbf{x}, \mathbf{y}} & q\left(\left\{1-\frac{a_{k} x_{k} b_{k} y_{k}}{\left(a_{k} x_{k}+\beta_{1}\right)\left(b_{k} y_{k}+\beta_{2}\right)}\right\}\right) \\
\text { s.t. } & \sum_{k=1}^{N_{b}} x_{k} \leq P_{1}, \quad x_{k} \geq 0, \quad k=1, \cdots, N_{b} \\
& \sum_{k=1}^{N_{b}} y_{k} \leq P_{2}, \quad y_{k} \geq 0, \quad k=1, \cdots, N_{b}
\end{array}
$$

where $\mathbf{x} \triangleq\left[x_{1}, \cdots, x_{N_{b}}\right]^{T}, \mathbf{y} \triangleq\left[y_{1}, \cdots, y_{N_{b}}\right]^{T}$, $\beta_{1}=\varepsilon_{1} \sum_{k=1}^{N_{b}} x_{k}\left[\mathbf{V}_{1,1}^{H} \mathbf{\Theta}_{1}^{T} \mathbf{V}_{1,1}\right]_{k, k}+1, \beta_{2}=\varepsilon_{2} \sum_{k=1}^{N_{b}} y_{k}$ $\left[\mathbf{V}_{2,1}^{H} \Theta_{2}^{T} \mathbf{V}_{2,1}\right]_{k, k}+1$. The optimal $\mathbf{x}$ and $\mathbf{y}$ in the problem (29)-(31) can be obtained by an iterative method developed in [4]. For any Schur-convex objective function $q$, since the optimal $\mathrm{E}_{\mathrm{H}_{1}, \mathrm{H}_{2}}[\mathbf{E}]$ has identical main diagonal elements, it can be shown similar to [4] that the optimal power loading vectors $\mathbf{x}$ and $\mathbf{y}$ are obtained by solving the problem (29)-(31) with $q=\sum_{k=1}^{N_{b}}\left[1-\frac{a_{k} x_{k} b_{k} y_{k}}{\left(a_{k} x_{k}+\beta_{1}\right)\left(b_{k} y_{k}+\beta_{2}\right)}\right]$.

For the case of $\boldsymbol{\Theta}_{i}=\tau_{i} \mathbf{I}_{N_{i}}, i=1,2$, we have $\alpha_{1}=$ $\tau_{1} \operatorname{tr}\left(\mathbf{F}_{1} \mathbf{F}_{1}^{H}\right)$, and $\alpha_{2}=\tau_{2} \operatorname{tr}\left(\mathbf{F}_{2}\left(\overline{\mathbf{H}}_{1} \mathbf{F}_{1} \mathbf{F}_{1}^{H} \overline{\mathbf{H}}_{1}^{H}+\alpha_{1} \boldsymbol{\Phi}_{1}+\right.\right.$ $\left.\left.\mathbf{I}_{N_{2}}\right) \mathbf{F}_{2}^{H}\right)$. Now we show that (9) is decreasing with respect to $\alpha_{1}$, i.e., if $\operatorname{tr}\left(\tilde{\mathbf{F}}_{1} \tilde{\mathbf{F}}_{1}^{H}\right) \leq \operatorname{tr}\left(\mathbf{F}_{1} \mathbf{F}_{1}^{H}\right)$, then $\mathrm{E}_{\mathrm{H}_{1}, \mathrm{H}_{2}}[\tilde{\mathbf{E}}] \succeq$ $\mathrm{E}_{\mathrm{H}_{1}, \mathrm{H}_{2}}[\mathbf{E}]$, where $\succeq$ denotes matrix positive-semidefiniteness, and $\tilde{\mathbf{E}}$ is obtained from (9) with $\tilde{\mathbf{F}}_{1}$. In fact, by introducing $\tilde{\mathbf{F}}_{1}=\alpha_{1}^{-\frac{1}{2}} \mathbf{F}_{1}$, (9) can be written as

$$
\mathrm{E}_{\mathrm{H}_{1}, \mathrm{H}_{2}}[\mathbf{E}]=\mathbf{I}_{N_{b}}-\tilde{\mathbf{F}}_{1}^{H} \overline{\mathbf{H}}_{1}^{H} \mathbf{F}_{2}^{H} \overline{\mathbf{H}}_{2}^{H} \overline{\mathbf{A}}^{-1} \overline{\mathbf{H}}_{2} \mathbf{F}_{2} \overline{\mathbf{H}}_{1} \tilde{\mathbf{F}}_{1}
$$

where

$$
\begin{aligned}
\overline{\mathbf{A}} \triangleq & \overline{\mathbf{H}}_{2} \mathbf{F}_{2}\left(\overline{\mathbf{H}}_{1} \tilde{\mathbf{F}}_{1} \tilde{\mathbf{F}}_{1}^{H} \overline{\mathbf{H}}_{1}^{H}+\boldsymbol{\Phi}_{1}+\alpha_{1}^{-1} \mathbf{I}_{N_{2}}\right) \mathbf{F}_{2}^{H} \overline{\mathbf{H}}_{2}^{H} \\
& +\tilde{\alpha}_{2} \mathbf{\Phi}_{2}+\alpha_{1}^{-1} \mathbf{I}_{N_{3}} \\
\tilde{\alpha}_{2} \triangleq & \operatorname{tr}\left(\mathbf{F}_{2}\left(\overline{\mathbf{H}}_{1} \tilde{\mathbf{F}}_{1} \tilde{\mathbf{F}}_{1}^{H} \overline{\mathbf{H}}_{1}^{H}+\boldsymbol{\Phi}_{1}+\alpha_{1}^{-1} \mathbf{I}_{N_{2}}\right) \mathbf{F}_{2}^{H}\right) .
\end{aligned}
$$

It can be clearly seen from (32)-(34) that for a given $\tilde{\mathbf{F}}_{1}$, $\mathrm{E}_{\mathrm{H}_{1}, \mathrm{H}_{2}}[\mathbf{E}]$ is a decreasing function of $\alpha_{1}$. It can be shown in a similar way to (32)-(34) that $\mathrm{E}_{\mathrm{H}_{1}, \mathrm{H}_{2}}[\mathbf{E}]$ also decreases with respect to $\alpha_{2}$. Thus, the optimal solution of $\mathbf{F}_{1}$ and $\mathbf{F}_{2}$ occurs 
at $\alpha_{1}=\tau_{1} P_{1}$ and $\alpha_{2}=\tau_{2} P_{2}$. Consequently, from (14)-(16), we find that $\tilde{\mathbf{U}}_{i}$ and $\tilde{\mathbf{V}}_{i}$ do not depend on $\mathbf{F}_{1}$ and $\mathbf{F}_{2}$.

Now the task is to find the $N_{b} \times N_{b}$ diagonal matrices $\boldsymbol{\Lambda}_{i}$, $i=1,2$. Substituting (17) back into (11)-(13), we have

$$
\begin{array}{rl}
\min _{\boldsymbol{\Lambda}_{1}, \boldsymbol{\Lambda}_{2}} & q\left(\mathbf{d}\left[\left[\mathbf{I}_{N_{b}}+\tilde{\boldsymbol{\Sigma}}_{1,1}^{2} \boldsymbol{\Lambda}_{1}^{2} \tilde{\boldsymbol{\Sigma}}_{2,1}^{2} \boldsymbol{\Lambda}_{2}^{2}\left(\tilde{\boldsymbol{\Sigma}}_{2,1}^{2} \boldsymbol{\Lambda}_{2}^{2}+\mathbf{I}_{N_{b}}\right)^{-1}\right]^{-1}\right]\right) \\
\text { s.t. } & \operatorname{tr}\left(\boldsymbol{\Lambda}_{2}^{2}\left(\boldsymbol{\Lambda}_{1}^{2} \tilde{\boldsymbol{\Sigma}}_{1,1}^{2}+\mathbf{I}_{N_{b}}\right)\right) \leq P_{2} \\
& \operatorname{tr}\left(\boldsymbol{\Lambda}_{1}^{2}\right) \leq P_{1}
\end{array}
$$

where $\tilde{\boldsymbol{\Sigma}}_{i, 1}$ is a diagonal matrix containing the largest $N_{b}$ singularvalues in $\tilde{\boldsymbol{\Sigma}}_{i}, i=1,2$. The problem (35)-(37) can be solved by the iterative method we just developed for solving the problem (29)-(31). Before moving to the next section, we would like to mention that as can be seen from (29)-(31) and (35)-(37), the proposed robust algorithm has the same computational complexity order as the algorithm developed in [4] which requires the exact CSI at all nodes. In other words, the improved robustness in performance is achieved without increasing the computational complexity.

\section{Robust MiMO Relay Design With EXACT CSI AT THE DESTINATION}

In some cases, channel estimation at the destination node can be accurate enough to be modelled as perfect (i.e., perfect CSI of $\mathbf{H}_{2}$ and $\mathbf{H}_{1}$ ), while the CSI available at the source and relay node is still imperfect due to feedback error/delay and quantization. In such case, the linear receiving matrix $\mathbf{W}$ can be designed to optimize the MSE matrix $\mathbf{E}$ in (2) as $\mathbf{W}=$ $\left[\mathbf{H}_{2} \mathbf{F}_{2}\left(\mathbf{H}_{1} \mathbf{F}_{1} \mathbf{F}_{1}^{H} \mathbf{H}_{1}^{H}+\mathbf{I}_{N_{2}}\right) \mathbf{F}_{2}^{H} \mathbf{H}_{2}^{H}+\mathbf{I}_{N_{3}}\right]^{-1} \mathbf{H}_{2} \mathbf{F}_{2} \mathbf{H}_{1} \mathbf{F}_{1}$. The resulting MSE matrix, defined as $\mathbf{E}_{0}$, is given as

$$
\begin{aligned}
\mathbf{E}_{0}= & {\left[\mathbf{I}_{N_{b}}+\mathbf{F}_{1}^{H} \mathbf{H}_{1}^{H} \mathbf{F}_{2}^{H} \mathbf{H}_{2}^{H}\left(\mathbf{H}_{2} \mathbf{F}_{2} \mathbf{F}_{2}^{H} \mathbf{H}_{2}^{H}+\mathbf{I}_{N_{3}}\right)^{-1}\right.} \\
& \left.\times \mathbf{H}_{2} \mathbf{F}_{2} \mathbf{H}_{1} \mathbf{F}_{1}\right]^{-1}
\end{aligned}
$$

Since the exact CSI of $\mathbf{H}_{1}$ and $\mathbf{H}_{2}$ is unknown at the node performing the optimization, we consider minimizing the objective function of $q\left(\mathbf{d}\left[\mathrm{E}_{\mathrm{H}_{1}, \mathrm{H}_{2}}\left[\mathbf{E}_{0}\right]\right]\right)$. However, it can be seen from (38) that it is intractable to obtain the expression of $\mathrm{E}_{\mathrm{H}_{1}, \mathrm{H}_{2}}\left[\mathbf{E}_{0}\right]$. To avoid the difficulty, in the following, we derive a lower bound of $\mathrm{E}_{\mathrm{H}_{1}, \mathrm{H}_{2}}\left[\mathbf{E}_{0}\right]$.

LEMMA 2 [11, Ch. 16]: For a matrix function $f(\mathbf{X})$ of random matrices $\mathbf{X}$ having finite expectation $\mathrm{E}[\mathbf{X}]$, if $f$ is matrix-convex, then $\mathrm{E}[f(\mathbf{X})] \succeq f(\mathrm{E}[\mathbf{X}])$.

It can be shown from [13] that $f(\mathbf{X})=\mathbf{X}^{-1}$ is a matrixconvex function of $\mathbf{X}$. Using Lemma 2 and (38), we find that for a fixed $\mathbf{H}_{2}$,

$$
\mathrm{E}_{\mathrm{H}_{1}}\left[\mathbf{E}_{0}\right] \succeq\left[\mathbf{I}_{N_{b}}+\mathbf{F}_{1}^{H} \mathrm{E}_{\mathrm{H}_{1}}\left[\mathbf{H}_{1}^{H} \mathbf{C}_{2} \mathbf{H}_{1}\right] \mathbf{F}_{1}\right]^{-1}
$$

where $\mathbf{C}_{2} \triangleq \mathbf{F}_{2}^{H} \mathbf{H}_{2}^{H}\left(\mathbf{H}_{2} \mathbf{F}_{2} \mathbf{F}_{2}^{H} \mathbf{H}_{2}^{H}+\mathbf{I}_{N_{3}}\right)^{-1} \mathbf{H}_{2} \mathbf{F}_{2}$. Let us define $\mathbf{C}_{1} \triangleq \mathbf{I}_{N_{b}}+\mathbf{F}_{1}^{H} \mathbf{H}_{1}^{H} \mathbf{H}_{1} \mathbf{F}_{1}$ and $\mathbf{C}_{3} \triangleq \mathbf{H}_{1} \mathbf{F}_{1}$, (38) can be written as

$$
\begin{aligned}
\mathbf{E}_{0}= & {\left[\mathbf{C}_{1}-\mathbf{C}_{3}^{H}\left(\mathbf{F}_{2}^{H} \mathbf{H}_{2}^{H} \mathbf{H}_{2} \mathbf{F}_{2}+\mathbf{I}_{N_{2}}\right)^{-1} \mathbf{C}_{3}\right]^{-1} } \\
= & \mathbf{C}_{1}^{-1}+\mathbf{C}_{1}^{-1} \mathbf{C}_{3}^{H}\left(\mathbf{F}_{2}^{H} \mathbf{H}_{2}^{H} \mathbf{H}_{2} \mathbf{F}_{2}+\mathbf{I}_{N_{2}}\right. \\
& \left.-\mathbf{C}_{3} \mathbf{C}_{1}^{-1} \mathbf{C}_{3}^{H}\right)^{-1} \mathbf{C}_{3} \mathbf{C}_{1}^{-1}
\end{aligned}
$$

where the matrix inversion lemma is applied to obtain (40). Using Lemma 2, it can be seen from (40) that for a given $\mathbf{H}_{1}$,

$$
\begin{aligned}
\mathrm{E}_{\mathrm{H}_{2}}\left[\mathbf{E}_{0}\right] \succeq \mathbf{C}_{1}^{-1} & +\mathbf{C}_{1}^{-1} \mathbf{C}_{3}^{H}\left(\mathbf{F}_{2}^{H} \mathrm{E}_{\mathrm{H}_{2}}\left[\mathbf{H}_{2}^{H} \mathbf{H}_{2}\right] \mathbf{F}_{2}\right. \\
& \left.+\mathbf{I}_{N_{2}}-\mathbf{C}_{3} \mathbf{C}_{1}^{-1} \mathbf{C}_{3}^{H}\right)^{-1} \mathbf{C}_{3} \mathbf{C}_{1}^{-1}
\end{aligned}
$$

From (39) and (41), we obtain $\mathbf{E}_{\mathrm{lb}}$, a lower bound of $\mathrm{E}_{\mathrm{H}_{1}, \mathrm{H}_{2}}\left[\mathbf{E}_{0}\right]$ as

$$
\begin{aligned}
\mathbf{E}_{\mathrm{lb}}= & {\left[\mathbf{I}_{N_{b}}+\mathbf{F}_{1}^{H} \mathrm{E}_{\mathrm{H}_{1}}\left[\mathbf { H } _ { 1 } ^ { H } \left[\mathbf{I}_{N_{2}}-\left(\mathbf{F}_{2}^{H} \mathrm{E}_{\mathrm{H}_{2}}\left[\mathbf{H}_{2}^{H} \mathbf{H}_{2}\right] \mathbf{F}_{2}\right.\right.\right.\right.} \\
& \left.\left.\left.\left.+\mathbf{I}_{N_{2}}\right)^{-1}\right] \mathbf{H}_{1}\right] \mathbf{F}_{1}\right]^{-1} \\
= & {\left[\mathbf{I}_{N_{b}}+\mathbf{F}_{1}^{H} \mathrm{E}_{\mathrm{H}_{1}}\left[\mathbf { H } _ { 1 } ^ { H } \left[\mathbf{I}_{N_{2}}-\left(\mathbf { F } _ { 2 } ^ { H } \left[\overline{\mathbf{H}}_{2}^{H} \overline{\mathbf{H}}_{2}\right.\right.\right.\right.\right.} \\
& \left.\left.\left.\left.\left.+\operatorname{tr}\left(\mathbf{\Phi}_{2}\right) \boldsymbol{\Theta}_{2}^{T}\right] \mathbf{F}_{2}+\mathbf{I}_{N_{2}}\right)^{-1}\right] \mathbf{H}_{1}\right] \mathbf{F}_{1}\right]^{-1} \\
= & {\left[\mathbf{I}_{N_{b}}+\mathbf{F}_{1}^{H}\left[\overline { \mathbf { H } } _ { 1 } ^ { H } \left[\mathbf{I}_{N_{2}}-\left(\mathbf { F } _ { 2 } ^ { H } \left[\overline{\mathbf{H}}_{2}^{H} \overline{\mathbf{H}}_{2}\right.\right.\right.\right.\right.} \\
& \left.\left.\left.\left.\left.+\operatorname{tr}\left(\mathbf{\Phi}_{2}\right) \boldsymbol{\Theta}_{2}^{T}\right] \mathbf{F}_{2}+\mathbf{I}_{N_{2}}\right)^{-1}\right] \mathbf{H}_{1}+\gamma \boldsymbol{\Theta}_{1}^{T}\right] \mathbf{F}_{1}\right]^{-1}
\end{aligned}
$$

where Lemma 1 is used to obtain (42) and (43) and $\gamma=$ $\operatorname{tr}\left(\boldsymbol{\Phi}_{1}\left[\mathbf{I}_{N_{2}}-\left(\mathbf{F}_{2}^{H}\left[\overline{\mathbf{H}}_{2}^{H} \overline{\mathbf{H}}_{2}+\operatorname{tr}\left(\boldsymbol{\Phi}_{2}\right) \mathbf{\Theta}_{2}^{T}\right] \mathbf{F}_{2}+\mathbf{I}_{N_{2}}\right)^{-1}\right]\right)$. Let us introduce $\hat{\mathbf{H}}_{2}^{H} \hat{\mathbf{H}}_{2} \triangleq \overline{\mathbf{H}}_{2}^{H} \overline{\mathbf{H}}_{2}+\operatorname{tr}\left(\boldsymbol{\Phi}_{2}\right) \boldsymbol{\Theta}_{2}^{T}, \tilde{\mathbf{F}}_{1} \triangleq\left(\boldsymbol{\Theta}_{1}^{T}\right)^{\frac{1}{2}} \mathbf{F}_{1}$, and $\hat{\mathbf{H}}_{1} \triangleq \overline{\mathbf{H}}_{1}\left(\boldsymbol{\Theta}_{1}^{T}\right)^{-\frac{1}{2}}$. Here $\boldsymbol{\Theta}_{1}^{T}=\left(\boldsymbol{\Theta}_{1}^{T}\right)^{\frac{H}{2}}\left(\boldsymbol{\Theta}_{1}^{T}\right)^{\frac{1}{2}}$. Then (43) can be rewritten as

$$
\begin{aligned}
\mathbf{E}_{\mathrm{lb}}=\left[\mathbf{I}_{N_{b}}+\tilde{\mathbf{F}}_{1}^{H} \hat{\mathbf{H}}_{1}^{H} \mathbf{F}_{2}^{H}\right. & \hat{\mathbf{H}}_{2}^{H}\left(\hat{\mathbf{H}}_{2} \mathbf{F}_{2} \mathbf{F}_{2}^{H} \hat{\mathbf{H}}_{2}^{H}+\mathbf{I}_{N_{3}}\right)^{-1} \\
& \left.\times \hat{\mathbf{H}}_{2} \mathbf{F}_{2} \hat{\mathbf{H}}_{1} \tilde{\mathbf{F}}_{1}+\gamma \tilde{\mathbf{F}}_{1}^{H} \tilde{\mathbf{F}}_{1}\right]^{-1}
\end{aligned}
$$

with $\gamma=\operatorname{tr}\left(\boldsymbol{\Phi}_{1} \mathbf{F}_{2}^{H} \hat{\mathbf{H}}_{2}^{H}\left(\hat{\mathbf{H}}_{2} \mathbf{F}_{2} \mathbf{F}_{2}^{H} \hat{\mathbf{H}}_{2}^{H}+\mathbf{I}_{N_{3}}\right)^{-1} \hat{\mathbf{H}}_{2} \mathbf{F}_{2}\right)$.

Using (44), (12), and (13), the robust relay design problem optimizing $q\left(\mathbf{d}\left[\mathbf{E}_{\mathrm{lb}}\right]\right)$ can be written as

$$
\begin{array}{rl}
\min _{\tilde{\mathbf{F}}_{1}, \mathbf{F}_{2}} & q\left(\mathbf { d } \left[\left[\mathbf{I}_{N_{b}}+\tilde{\mathbf{F}}_{1}^{H} \hat{\mathbf{H}}_{1}^{H} \mathbf{F}_{2}^{H} \hat{\mathbf{H}}_{2}^{H}\left(\hat{\mathbf{H}}_{2} \mathbf{F}_{2} \mathbf{F}_{2}^{H} \hat{\mathbf{H}}_{2}^{H}+\mathbf{I}_{N_{3}}\right)^{-1}\right.\right.\right. \\
& \left.\left.\left.\times \hat{\mathbf{H}}_{2} \mathbf{F}_{2} \hat{\mathbf{H}}_{1} \tilde{\mathbf{F}}_{1}+\gamma \tilde{\mathbf{F}}_{1}^{H} \tilde{\mathbf{F}}_{1}\right]^{-1}\right]\right) \\
\text { s.t. } & \operatorname{tr}\left(\mathbf { F } _ { 2 } \left(\hat{\mathbf{H}}_{1} \tilde{\mathbf{F}}_{1} \tilde{\mathbf{F}}_{1}^{H} \hat{\mathbf{H}}_{1}^{H}+\operatorname{tr}\left(\tilde{\mathbf{F}}_{1} \tilde{\mathbf{F}}_{1}^{H}\right) \boldsymbol{\Phi}_{1}\right.\right. \\
& \left.\left.+\mathbf{I}_{N_{2}}\right) \mathbf{F}_{2}^{H}\right) \leq P_{2} \\
& \operatorname{tr}\left(\tilde{\mathbf{F}}_{1}^{H}\left(\boldsymbol{\Theta}_{1}^{T}\right)^{-\frac{H}{2}}\left(\boldsymbol{\Theta}_{1}^{T}\right)^{-\frac{1}{2}} \tilde{\mathbf{F}}_{1}\right) \leq P_{1} .
\end{array}
$$

In contrast to the case of imperfect CSI at all nodes discussed in Section III, it is very difficult to find the optimal structure of $\tilde{\mathbf{F}}_{1}$ and $\mathbf{F}_{2}$ as the solution to the problem (45)-(47). Inspired by the robust design in Section III, we adopt a (sub)optimal structure of $\tilde{\mathbf{F}}_{1}$ and $\mathbf{F}_{2}$ as $\tilde{\mathbf{F}}_{1}=\hat{\mathbf{V}}_{1,1} \boldsymbol{\Lambda}_{1}, \mathbf{F}_{2}=\hat{\mathbf{V}}_{2,1} \boldsymbol{\Lambda}_{2} \hat{\mathbf{U}}_{1,1}^{H}$, where $\hat{\mathbf{H}}_{i}=\hat{\mathbf{U}}_{i} \hat{\boldsymbol{\Sigma}}_{i} \hat{\mathbf{V}}_{i}^{H}, i=1,2$, is the SVD of $\hat{\mathbf{H}}_{i}, \hat{\mathbf{U}}_{i, 1}$ and $\hat{\mathbf{V}}_{i, 1}, i=1,2$, contain $N_{b}$ columns in $\hat{\mathbf{U}}_{i}$ and $\hat{\mathbf{V}}_{i}$ associated with the largest $N_{b}$ singularvalues, respectively. Now the robust relay design problem boils down to the optimization 
of the power loading matrices $\Lambda_{1}$ and $\boldsymbol{\Lambda}_{2}$, which is given by

$$
\begin{array}{rl}
\min _{\boldsymbol{\lambda}_{1}, \boldsymbol{\lambda}_{2}} & q\left(\left\{\left(1+\frac{\hat{\sigma}_{1, k}^{2} \lambda_{1, k}^{2} \hat{\sigma}_{2, k}^{2} \lambda_{2, k}^{2}}{\hat{\sigma}_{2, k}^{2} \lambda_{2, k}^{2}+1}+\gamma \lambda_{1, k}^{2}\right)^{-1}\right\}\right) \\
\text { s.t. } & \sum_{k=1}^{N_{b}} \lambda_{2, k}^{2}\left(\hat{\sigma}_{1, k}^{2} \lambda_{1, k}^{2}+m_{k} \sum_{j=1}^{N_{b}} \lambda_{1, j}^{2}+1\right) \leq P_{2} \\
& \sum_{k=1}^{N_{b}} d_{k} \lambda_{1, k}^{2} \leq P_{1} \\
& \lambda_{1, k} \geq 0, \quad \lambda_{2, k} \geq 0, \quad k=1, \cdots, N_{b}
\end{array}
$$

where for $i=1,2, \lambda_{i, k}$ and $\hat{\sigma}_{i, k}, k=1, \cdots, N_{b}$, are the $k$ th largest main diagonal elements of $\boldsymbol{\Lambda}_{i}$ and $\hat{\boldsymbol{\Sigma}}_{i}$, respectively, $\gamma=\sum_{k=1}^{N_{b}} \frac{\hat{\sigma}_{2, k}^{2} \lambda_{2, k}^{2} m_{k}}{\hat{\sigma}_{2, k}^{2} \lambda_{2, k}^{2}+1}, m_{k}$ is the $k$ th main diagonal element of $\mathbf{M} \triangleq \hat{\mathbf{U}}_{1,1}^{H} \boldsymbol{\Phi}_{1} \hat{\mathbf{U}}_{1,1}$, and $d_{k}$ is the $k$ th main diagonal element of $\mathbf{D} \triangleq \hat{\mathbf{V}}_{1,1}^{H}\left(\boldsymbol{\Theta}_{1}^{T}\right)^{-\frac{H}{2}}\left(\boldsymbol{\Theta}_{1}^{T}\right)^{-\frac{1}{2}} \hat{\mathbf{V}}_{1,1}$.

By introducing $x_{k} \triangleq \lambda_{1, k}^{2}, a_{k} \triangleq \hat{\sigma}_{1, k}^{2}, b_{k} \triangleq \hat{\sigma}_{2, k}^{2}$, $y_{k} \triangleq \lambda_{2, k}^{2}\left(\hat{\sigma}_{1, k}^{2} \lambda_{1, k}^{2}+m_{k} \sum_{j=1}^{N_{b}} \lambda_{1, j}^{2}+1\right), k=1, \cdots, N_{b}$, the problem (48)-(51) can be equivalently written as

$$
\begin{array}{ll}
\min _{\mathbf{x}, \mathbf{y}} & q\left(\left\{\left(1+\frac{a_{k} b_{k} x_{k} y_{k}}{a_{k} x_{k}+b_{k} y_{k}+m_{k} \sum_{j=1}^{N_{b}} x_{j}+1}\right.\right.\right. \\
& \left.\left.\left.+\gamma x_{k}\right)^{-1}\right\}\right) \\
\text { s.t. } & \sum_{k=1}^{N_{b}} y_{k} \leq P_{2}, \quad y_{k} \geq 0, \quad k=1, \cdots, N_{b} \\
& \sum_{k=1}^{N_{b}} d_{k} x_{k} \leq P_{1}, \quad x_{k} \geq 0, \quad k=1, \cdots, N_{b}
\end{array}
$$

where we have now $\gamma=\sum_{k=1}^{N_{b}} \frac{b_{k} y_{k} m_{k}}{a_{k} x_{k}+b_{k} y_{k}+m_{k} \sum_{j=1}^{N_{b}} x_{j}+1}$. Similar to the problem (29)-(31), the problem (52)-(54) can be efficiently solved by iteratively updating $\mathbf{x}$ and $\mathbf{y}$.

\section{NumERICAL EXAMPLES}

In this section, we study the performance of the proposed robust source and relay matrices through numerical simulations. In the simulations, the estimated channel matrices $\overline{\mathbf{H}}_{1}$ and $\overline{\mathbf{H}}_{2}$ have i.i.d. complex Gaussian entries with zeromean and variances $\sigma_{i}^{2} / N_{i}$ for $\overline{\mathbf{H}}_{i}, i=1,2$. We define $\mathrm{SNR}_{i}=\sigma_{i}^{2} P_{i} N_{i+1} / N_{i}$ as the signal-to-noise ratio (SNR) for the $i$ th hop, $i=1,2$. In all simulations, we set $N_{i}=4$, $i=1,2,3, N_{b}=3, \mathrm{SNR}_{2}=20 \mathrm{~dB}$, and all simulation results are averaged over 1000 independent realizations of the true channel matrices $\mathbf{H}_{1}$ and $\mathbf{H}_{2}$. We will consider the following five MIMO relay algorithms.

- Non-RB (Imp. CSID): The algorithm proposed in [4] using the imperfect CSI at all nodes.

- Non-RB (Exact CSID): The algorithm developed in [4] with the exact CSI at the destination node.

- RB (Imp. CSID): The robust algorithm with imperfect CSI at the destination node developed in Section III with $q=\operatorname{tr}\left(\mathrm{E}_{\mathrm{H}_{1}, \mathrm{H}_{2}}[\mathbf{E}]\right)$.
- RB (Exact CSID): The robust algorithm with the exact CSI at the destination node developed in Section IV with $q=\operatorname{tr}\left(\mathbf{E}_{\mathrm{lb}}\right)$.

- Exact CSI (All nodes): The MIMO relay algorithm proposed in [4] using the exact CSI at all nodes.

In the first example, the true channel matrices are modelled as (3) with $\boldsymbol{\Theta}_{i}=\mathbf{I}_{N_{i}}, i=1,2$, and $\boldsymbol{\Phi}_{i}, i=1,2$, are defined as Toeplitz matrices [5]-[9] with elements given by $\left[\boldsymbol{\Phi}_{1}\right]_{m, n}=$ $0.8^{|m-n|}$ and $\left[\boldsymbol{\Phi}_{2}\right]_{m, n}=0.9^{|m-n|}$, respectively. Fig. 1 shows the MSE performance of four algorithms versus $\mathrm{SNR}_{1}$. It can be seen that for a MIMO relay system with imperfect CSI at the destination node, the MSE produced by the non-robust relay algorithm increases with $\mathrm{SNR}_{1}$. This is due to the fact that the mismatch between $\mathbf{H}_{i}$ and $\overline{\mathbf{H}}_{i}$ is not considered by the non-robust relay algorithm. The robust algorithm developed in Section III has a much better MSE performance compared with the non-robust algorithm. But due to the missing of the exact CSI at the destination node, it still yields a high errorfloor. With the exact CSI at the destination node, the robust relay algorithm proposed in Section IV yields a similar slope of decreasing MSE with respect to $\mathrm{SNR}_{1}$ as the relay scheme with the exact CSI at all nodes.

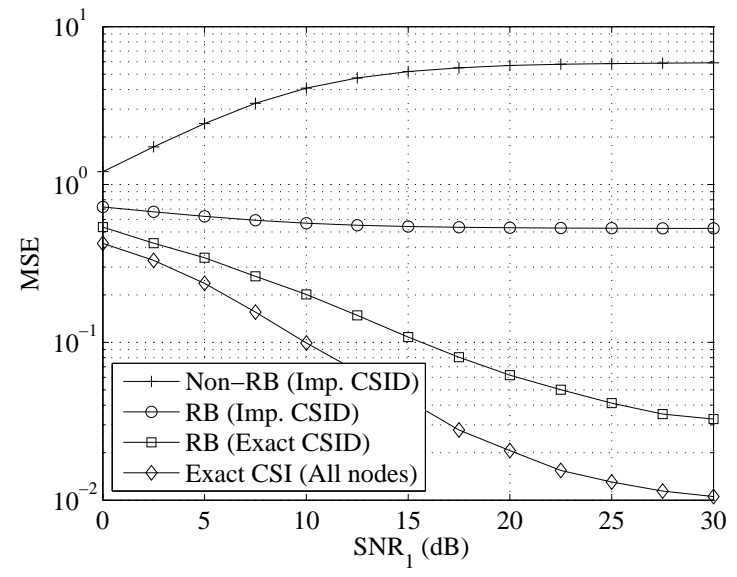

Fig. 1. Example 1: MSE versus $\mathrm{SNR}_{1}$.

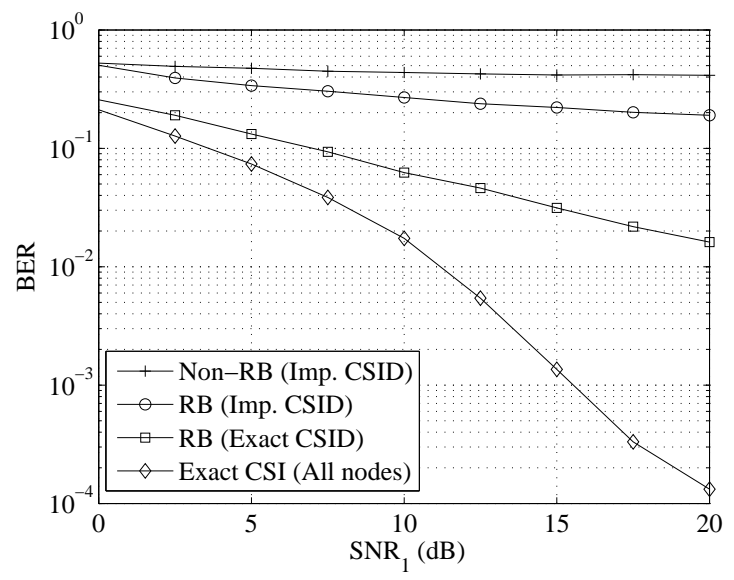

Fig. 2. Example 1: BER versus $\mathrm{SNR}_{1}$. 
Fig. 2 shows the BER performance of four relay algorithms versus $\mathrm{SNR}_{1}$. The QPSK constellations are used to modulate the source symbols. We observe that when the exact CSI is not available at the destination node, the non-robust algorithm has a BER close to 0.5 over the whole $\mathrm{SNR}_{1}$ range. The robust relay algorithm has a better performance in this case. With the exact CSI at the destination node, the robust relay algorithm proposed in Section IV further improves the system BER performance. But at large $\mathrm{SNR}_{1}$, there is still some gap between the BER of the relay system with the exact CSI at all nodes and the relay system with imperfect CSI at the source and relay nodes.

In the second example, the true channel matrices are modelled as (3) with $\left[\boldsymbol{\Theta}_{1}\right]_{m, n}=0.7^{|m-n|},\left[\boldsymbol{\Theta}_{2}\right]_{m, n}=0.8^{|m-n|}$, $\left[\boldsymbol{\Phi}_{1}\right]_{m, n}=0.6^{|m-n|}$, and $\left[\boldsymbol{\Phi}_{2}\right]_{m, n}=0.7^{|m-n|}$. As mentioned in Section III, the explicit structure of the optimal robust $\mathbf{F}_{1}$ and $\mathbf{F}_{2}$ is difficult to obtain when the destination node has the imperfect CSI. Thus, we compare the performance of three relay systems with the exact CSI at the destination. Fig. 3 shows the MSE performance of three algorithms versus $\mathrm{SNR}_{1}$, while Fig. 4 displaces the system BER produced by three algorithms versus $\mathrm{SNR}_{1}$. Similar to Figs. 1 and 2, we observe that the robust relay algorithm developed in Section IV has improved MSE and BER performance than the non-robust algorithm.

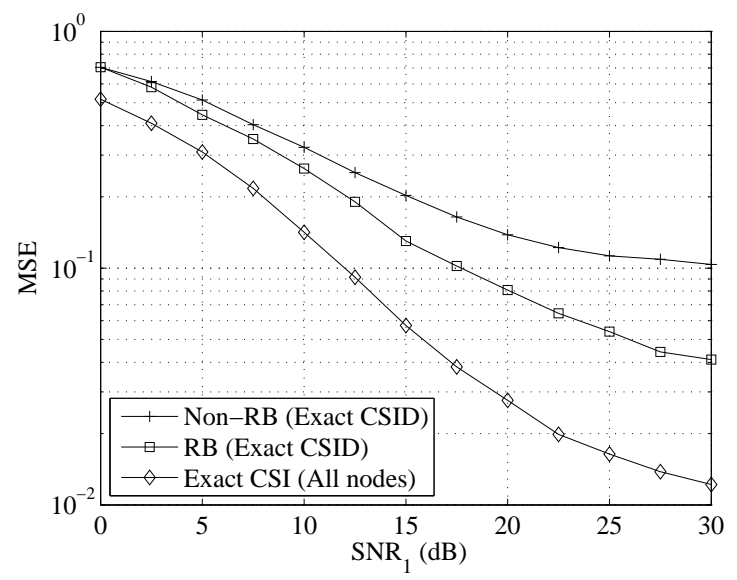

Fig. 3. Example 2: MSE versus $\mathrm{SNR}_{1}$.

\section{CONCLusions}

We have addressed two imperfect CSI scenarios in linear non-regenerative MIMO relay communications. For each case, we have developed statistically robust source and relay matrices for most commonly used MIMO system design criteria. Simulation results show an improved robustness of the proposed algorithms against CSI errors.

\section{REFERENCES}

[1] X. Tang and Y. Hua, "Optimal design of non-regenerative MIMO wireless relays," IEEE Trans. Wireless Commun., vol. 6, pp. 1398-1407, Apr. 2007.

[2] I. Hammerström and A. Wittneben, "Power allocation schemes for amplify-and-forward MIMO-OFDM relay links," IEEE Trans. Wireless Commun., vol. 6, pp. 2798-2802, Aug. 2007.

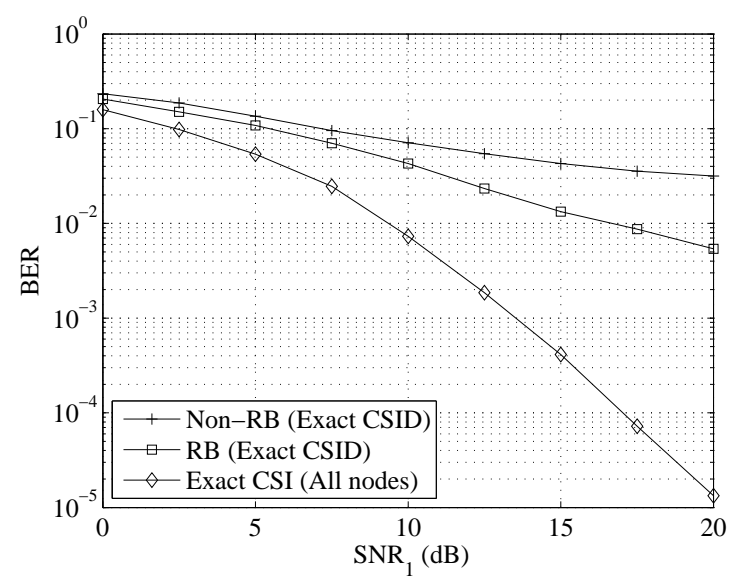

Fig. 4. Example 2: BER versus $\mathrm{SNR}_{1}$.

[3] A. S. Behbahani, R. Merched, and A. M. Eltawil, "Optimizations of a MIMO relay network,” IEEE Trans. Signal Process., vol. 56, pp. 50625073, Oct. 2008.

[4] Y. Rong, X. Tang, and Y. Hua, "A unified framework for optimizing linear non-regenerative multicarrier MIMO relay communication systems," IEEE Trans. Signal Process., vol. 57, pp. 4837-4851, Dec. 2009.

[5] C. Xing, S. Ma, and Y.-C. Wu, "Bayesian robust linear transceiver design for dual-hop amplify-and-forward MIMO relay systems," in Proc. IEEE Globecom, Honolulu, HI, Nov. 2009.

[6] C. Xing, S. Ma, Y.-C. Wu, T.-S. Ng, and H. V. Poor, "Linear transceiver design for amplify-and-forward MIMO relay systems under channel uncertainties," in Proc. IEEE WCNC, Sydney, Australia, Apr. 2010.

[7] C. Xing, S. Ma, and Y.-C. Wu, "Robust joint design of linear relay precoder and destination equalizer for dual-hop amplify-and-forward MIMO relay systems," IEEE Trans. Signal Process., vol. 58, pp. 22732283, Apr. 2010.

[8] B. K. Chalise and L. Vandendorpe, "Joint linear processing for an amplify-and-forward MIMO relay channel with imperfect channel state information," EURASIP J. Advances Signal Process. vol. 2010, Article ID 640186, 13 pages.

[9] C. Xing, S. Ma, Y.-C. Wu, and T.-S. Ng, "Transceiver design for dual-hop non-regenerative MIMO-OFDM relay systems under channel uncertainties," IEEE Trans. Signal Process., vol. 58, pp. 6325-6339, Dec. 2010.

[10] Y. Rong, "Robust design for linear non-regenerative MIMO relays," in Proc. Australian Communications Theory Workshop, Canberra, Australia, Feb. 2-5, 2010, pp. 87-92.

[11] A. W. Marshall and I. Olkin, Inequalities: Theory of Majorization and Its Applications. Academic Press, 1979.

[12] A. Gupta and D. Nagar, Matrix Variate Distributions. London, U.K.: Chapman \& Hall/CRC, 2000.

[13] E. Jorswieck and H. Boche, Majorization and Matrix-Monotone Functions in Wireless Communications. Now Publishers, 2007. 